\title{
A Short Proof of Stirling's Formula
}

\author{
Hongwei Lou
}

\begin{abstract}
By changing variables in a suitable way and using dominated convergence methods, this note gives a short proof of Stirling's formula and its refinement.
\end{abstract}

\section{Introduction.}

This note provides a short proof of the well-known Stirling's formula

$$
\Gamma(s+1)=\left(\frac{s}{e}\right)^{s} \sqrt{2 \pi s}(1+o(1)), \quad \text { as } s \rightarrow+\infty,
$$

where $\Gamma$ is Euler's Gamma function

$$
\Gamma(s):=\int_{0}^{+\infty} t^{s-1} e^{-t} d t, \quad \text { for } s>0 .
$$

There are many alternative proofs of Stirling's formula in the literature. For instance, one can find proofs given by P. Diaconis, D. Freedman [1, J. M. Partin [2] and M. Pinsky [3]. The proof presented below has some common elements with the ones in [1] and 2, but appears much simpler in that it also gives a short and clear way to refine Stirling's formula (cf. (18) and (20)).

\section{Proof of Stirling's formula.}

Let $s>0$. Changing variables $(t=s(x+1))$ leads to

$$
\Gamma(s+1)=\int_{0}^{+\infty} t^{s} e^{-t} d t=\frac{s^{s+1}}{e^{s}} \int_{-1}^{+\infty} e^{-s g(x)} d x,
$$

where

$$
g(x):=x-\ln (1+x), \quad \text { for } x \in(-1,+\infty) .
$$

One can easily see that $g(x)$ is strictly convex in $(-1,+\infty)$, admitting its minimum 0 at $x=0$. Moreover, $\lim _{x \rightarrow-1^{+}} g(x)=\lim _{x \rightarrow+\infty} g(x)=+\infty$. 
Let $v(x)=\sqrt{g(x)} \operatorname{sgn} x$ and $x=f(v)$ be the inverse function of $v(x)$. By a change of variables $\left(x=f\left(\frac{u}{\sqrt{s}}\right)\right)$, one has

$$
\begin{aligned}
\Gamma(s+1) & =\left(\frac{s}{e}\right)^{s} \sqrt{s} \int_{-\infty}^{+\infty} 2\left(\frac{\frac{u}{\sqrt{s}}}{f\left(\frac{u}{\sqrt{s}}\right)}+\frac{u}{\sqrt{s}}\right) e^{-u^{2}} d u \\
& =\left(\frac{s}{e}\right)^{s} \sqrt{s} \int_{0}^{+\infty} 2\left(y\left(\frac{u}{\sqrt{s}}\right)+y\left(-\frac{u}{\sqrt{s}}\right)\right) e^{-u^{2}} d u
\end{aligned}
$$

where

$$
y(v)= \begin{cases}\frac{v}{f(v)}, & v \neq 0, \\ \frac{\sqrt{2}}{2}, & v=0 .\end{cases}
$$

Since $f(v)$ is continuous and

$$
\lim _{v \rightarrow 0} y(v)=\lim _{v \rightarrow 0} \frac{v}{f(v)}=\lim _{x \rightarrow 0} \frac{\sqrt{x-\ln (1+x)}}{|x|}=\frac{\sqrt{2}}{2},
$$

it follows that $y(v)$ is continuous. Note that $f(v)$ is strictly increasing on $(-\infty,+\infty)$ with $f(0)=0$, the equations (6) and (7) imply that

$$
0<y(v) \leq C(|v|+1), \quad \text { for all } v \in(-\infty,+\infty)
$$

for some constant $C>0$. Thus, one deduces from (8) and the continuity of $y(v)$ at $v=0$ that

$$
\lim _{s \rightarrow+\infty} \int_{0}^{+\infty} 2\left(y\left(\frac{u}{\sqrt{s}}\right)+y\left(-\frac{u}{\sqrt{s}}\right)\right) e^{-u^{2}} d u=\int_{0}^{+\infty} 4 y(0) e^{-u^{2}} d u=\sqrt{2 \pi}
$$

which yields Stirling's formula (11).

More precisely, we can prove (9) by virtue of the following estimate:

$$
\begin{aligned}
& \left|\int_{0}^{+\infty}\left(y\left(\frac{u}{\sqrt{s}}\right)+y\left(-\frac{u}{\sqrt{s}}\right)-2 y(0)\right) e^{-u^{2}} d u\right| \\
\leq & \int_{\sqrt[4]{s}}^{+\infty}\left(2 C\left(\frac{u}{\sqrt{s}}+1\right)+\sqrt{2}\right) e^{-u^{2}} d u+\int_{0}^{\sqrt[4]{s}} 2 \omega\left(\frac{1}{\sqrt[4]{s}}\right) e^{-u^{2}} d u \\
\leq & \int_{\sqrt[4]{s}}^{+\infty}(4 C+2) u e^{-u^{2}} d u+\int_{0}^{+\infty} 2 \omega\left(\frac{1}{\sqrt[4]{s}}\right) e^{-u^{2}} d u, \quad \text { for all } s \geq 1,(10)
\end{aligned}
$$

where

$$
\omega(r):=\max _{|v| \leq r}|y(v)-y(0)|, \quad \text { for } r>0,
$$

satisfying $\lim _{r \rightarrow 0^{+}} \omega(r)=0$. 


\section{Refinement of Stirling's formula.}

To refine Stirling's formula, we mention that

$$
G(x):= \begin{cases}\frac{g(x)}{x^{2}}, & x \neq 0 \\ \frac{1}{2}, & x=0\end{cases}
$$

is a positive smooth function in $(-1,+\infty)$ and $y(v)$ is the unique solution of

$$
y(v)=\sqrt{G\left(\frac{v}{y(v)}\right)}, \quad \text { for } v \in(-\infty,+\infty) .
$$

Then the smoothness of $y(v)$ follows easily from the smoothness of $\sqrt{G}$ and

$$
\left.\left[\frac{\partial}{\partial y}\left(y-\sqrt{G\left(\frac{v}{y}\right)}\right)\right]\right|_{y=y(v)}=\frac{1}{2(1+f(v)) y^{2}(v)}>0 .
$$

Denote by $\sum_{k=0}^{\infty} a_{k} v^{k}$ the MacLaurin's series of $y(v)$. Thus, we have

$$
y(v)+y(-v)=\sum_{k=0}^{n} 2 a_{2 k} v^{2 k}+o\left(v^{2 n}\right), \quad \text { for } v \rightarrow 0 .
$$

Thus, similar to (8), there exist constants $C_{n}>0$ (for $\left.n=1,2, \ldots\right)$ such that

$$
\left|y(v)+y(-v)-\sum_{k=0}^{n} 2 a_{2 k} v^{2 k}\right| \leq C_{n} v^{2 n}, \quad \text { for all } v \in(-\infty, \infty) .
$$

While analogous to (10), we have that for any $s \geq 1$,

$$
\begin{aligned}
& \left|\int_{0}^{+\infty}\left(y\left(\frac{u}{\sqrt{s}}\right)+y\left(-\frac{u}{\sqrt{s}}\right)-\sum_{k=0}^{n} 2 a_{2 k} \frac{u^{2 k}}{s^{k}}\right) e^{-u^{2}} d u\right| \\
& \leq \frac{1}{s^{n}}\left[C_{n} \int_{\sqrt[4]{s}}^{+\infty} u^{2 n} e^{-u^{2}} d u+\omega_{n}\left(\frac{1}{\sqrt[4]{s}}\right) \int_{0}^{+\infty} u^{2 n} e^{-u^{2}} d u\right]
\end{aligned}
$$

with

$$
\omega_{n}(r):=\sup _{0<v \leq r} \frac{1}{v^{2 n}}\left|y(v)+y(-v)-\sum_{k=0}^{n} a_{2 k} v^{2 k}\right|, \quad \text { where } r>0 .
$$

As a consequence of (17) and $\lim _{r \rightarrow 0^{+}} \omega_{n}(r)=0$,

$$
\begin{aligned}
\Gamma(s+1) & =\left(\frac{s}{e}\right)^{s} \sqrt{s}\left[\int_{0}^{+\infty} \sum_{k=0}^{n} 4 a_{2 k} \frac{u^{2 k}}{s^{k}} e^{-u^{2}} d u+o\left(\frac{1}{s^{n}}\right)\right] \\
& =\left(\frac{s}{e}\right)^{s} \sqrt{s}\left[\sum_{k=0}^{n} 2 a_{2 k} \Gamma\left(k+\frac{1}{2}\right) \frac{1}{s^{k}}+o\left(\frac{1}{s^{n}}\right)\right], \quad \text { as } s \rightarrow+\infty
\end{aligned}
$$


For example, for $n=5$, a direct calculation shows that

$$
\begin{aligned}
& a_{0}=\frac{\sqrt{2}}{2}, a_{2}=\frac{\sqrt{2}}{12}, a_{4}=\frac{\sqrt{2}}{432}, a_{6}=-\frac{139 \sqrt{2}}{194400}, \\
& a_{8}=-\frac{571 \sqrt{2}}{32659200}, a_{10}=\frac{163879 \sqrt{2}}{12345177600} .
\end{aligned}
$$

It turns out that (18) becomes

$$
\begin{aligned}
\Gamma(s+1)= & \left(\frac{s}{e}\right)^{s} \sqrt{2 \pi s}\left[1+\frac{1}{12 s}+\frac{1}{288 s^{2}}-\frac{139}{51840 s^{3}}\right. \\
& \left.-\frac{571}{2488320 s^{4}}+\frac{163879}{209018880 s^{5}}+o\left(\frac{1}{s^{5}}\right)\right], \quad \text { as } s \rightarrow+\infty .
\end{aligned}
$$

Acknowledgments. This work was supported in part by NSFC (No. 61074047).

\section{References}

[1] P. Diaconis, D. Freedman, An elementary proof of Stirling's formula, Amer. Math. Monthly 93 (1986) 123-125.

[2] J. M. Partin, A very short proof of Stirling's formula, Amer. Math. Monthly 96 (1989) 41-42.

[3] M. Pinsky, Stirling's formula via the Poisson distribution, Amer. Math. Monthly 114 (2007) 256-258.

School of Mathematical Sciences, Fudan University, Shanghai 200433, China hwlou@fudan.edu.cn 


\section{Appendix: Methods to Calculate $a_{n}$.}

First, we have $a_{0}=\frac{\sqrt{2}}{2}$.

\section{Method I.}

$$
\begin{aligned}
a_{n} & =\lim _{v \rightarrow 0} \frac{y(v)-\sum_{k=0}^{n-1} a_{k} v^{k}}{v^{n}}=\lim _{v \rightarrow 0^{+}} \frac{y(v)-\sum_{k=0}^{n-1} a_{k} v^{k}}{v^{n}} \\
& =\lim _{v \rightarrow 0^{+}} \frac{\frac{v}{f(v)}-\sum_{k=0}^{n-1} a_{k} v^{k}}{v^{n}} \\
& =\lim _{x \rightarrow 0^{+}} \frac{\frac{\sqrt{x-\ln (1+x)}}{x}-\sum_{k=0}^{n-1} a_{k}(x-\ln (1+x))^{\frac{k}{2}}}{(x-\ln (1+x))^{\frac{n}{2}}}, \quad n \geq 1 . \\
& =\lim _{x \rightarrow 0^{+}} \frac{2^{\frac{n}{2}}\left(\frac{\sqrt{x-\ln (1+x)}}{x}-\sum_{k=0}^{n-1} a_{k}(x-\ln (1+x))^{\frac{k}{2}}\right)}{x^{n}}, \quad n
\end{aligned}
$$

Method II. It follows from

$$
v^{2}=\frac{v}{y(v)}-\ln \left(1+\frac{v}{y(v)}\right)
$$

that

$$
\frac{v}{v+y}\left(\frac{v}{y}\right)^{\prime}=2 v
$$

Then

$$
v y^{\prime}=y-2 y^{2}-2 v y^{2} .
$$

For two smooth (not necessary analytic) functions $\varphi(t)$ and $\psi(t)$, the MacLaurin's series of $\varphi(t) \psi(t)$ is the Cauchy product of the MacLaurin's series of $\varphi(t)$ and that of $\psi(t)$. Then we can establish that

$$
\begin{aligned}
a_{n}= & \text { the coefficient of } v^{n} \text { in the expansion of } \\
& -\frac{2}{n+2}\left[\left(\sum_{k=0}^{n-1} a_{k} v^{k}\right)^{3}+v\left(\sum_{k=0}^{n-1} a_{k} v^{k}\right)^{2}\right] \\
= & -\frac{2}{n+2}\left[\sum_{\substack{k+j+l=n \\
0 \leq k, j, l<n}} a_{k} a_{j} a_{l}+\sum_{k+j=n-1} a_{k} a_{j}\right], \quad n \geq 1 .
\end{aligned}
$$


More precisely, for $n \geq 1$, we have

$$
\begin{aligned}
& a_{0}-\sum_{k=1}^{n}(k-1) a_{k} v^{k} \\
= & 2\left(\sum_{k=0}^{n} a_{k} v^{k}\right)^{3}+2 v\left(\sum_{k=0}^{n} a_{k} v^{k}\right)^{2}+o\left(v^{n}\right) \\
= & 6 a_{0}^{2} a_{n} v^{n}+2\left(\sum_{k=0}^{n-1} a_{k} v^{k}\right)^{3}+2 v\left(\sum_{k=0}^{n-1} a_{k} v^{k}\right)^{2}+o\left(v^{n}\right) \\
= & 3 a_{n} v^{n}+2\left(\sum_{k=0}^{n-1} a_{k} v^{k}\right)^{3}+2 v\left(\sum_{k=0}^{n-1} a_{k} v^{k}\right)^{2}+o\left(v^{n}\right), \quad(v \rightarrow 0) .
\end{aligned}
$$

That is,

$$
a_{0}-\sum_{k=1}^{n}(k+2) a_{k} v^{k}=2\left(\sum_{k=0}^{n-1} a_{k} v^{k}\right)^{3}+2 v\left(\sum_{k=0}^{n-1} a_{k} v^{k}\right)^{2}+o\left(v^{n}\right), \quad(v \rightarrow 0) .
$$

Therefore,

$$
\begin{array}{r}
a_{n}=\text { the coefficient of } v^{n} \text { in the expansion of } \\
-\frac{2}{n+2}\left[\left(\sum_{k=0}^{n-1} a_{k} v^{k}\right)^{3}+v\left(\sum_{k=0}^{n-1} a_{k} v^{k}\right)^{2}\right] .
\end{array}
$$

The advantage of Method I is that the convergence of the limit in calculating $a_{n+1}$ depends on the correctness of $a_{0}, a_{1}, \ldots, a_{n}$. Thus, we can know whether $a_{n}$ is correct or not when we calculate $a_{n+1}$.

The advantage of Method II is that it brings possibility to calculate $a_{n}$ without the aid of computer. 
We have:

$$
\begin{aligned}
& a_{0}=\frac{1}{2} \sqrt{2}, \quad a_{1}=-\frac{1}{3}, \\
& a_{2}=\frac{1}{12} \sqrt{2}, \quad a_{3}=-\frac{4}{135} \\
& a_{4}=\frac{1}{432} \sqrt{2}, \quad a_{5}=\frac{4}{2835}, \\
& a_{6}=-\frac{139}{194400} \sqrt{2}, \quad a_{7}=\frac{8}{25515}, \\
& a_{8}=-\frac{571}{32659200} \sqrt{2}, \quad a_{9}=-\frac{1124}{37889775}, \\
& a_{10}=\frac{163879}{12345177600} \sqrt{2}, \quad a_{11}=-\frac{41768}{7388506125}, \\
& a_{12}=\frac{5246819}{24443451648000} \sqrt{2}, \quad a_{13}=\frac{43672}{66496555125} \\
& a_{14}=-\frac{534703531}{1906589228544000} \sqrt{2}, \quad a_{15}=\frac{1459313264}{12463116844303125}, \\
& a_{16}=-\frac{4483131259}{1372744244551680000} \sqrt{2}, \quad a_{17}=-\frac{10603947212}{710397660125278125}, \\
& a_{18}=\frac{432261921612371}{69309856907414323200000} \sqrt{2}, \quad a_{19}=-\frac{49374413464}{19180736823382509375}, \\
& a_{20}=\frac{6232523202521089}{110618531624233259827200000} \sqrt{2} \\
& \Gamma(s+1)=\left(\frac{s}{e}\right)^{s} \sqrt{2 \pi s}\left[1+\frac{1}{12 s}+\frac{1}{288 s^{2}}-\frac{139}{51840 s^{3}}-\frac{571}{2488320 s^{4}}\right. \\
& +\frac{163879}{209018880 s^{5}}+\frac{5246819}{75246796800 s^{6}}-\frac{534703531}{902961561600 s^{7}} \\
& -\frac{4483131259}{86684309913600 s^{8}}+\frac{432261921612371}{514904800886784000 s^{9}} \\
& \left.+\frac{6232523202521089}{86504006548979712000 s^{10}}+o\left(\frac{1}{s^{10}}\right)\right], \quad \text { as } s \rightarrow+\infty \text {. }
\end{aligned}
$$

